\title{
Optimal Homotopy Asymptotic Method for Solving Delay Differential Equations
}

\author{
N. Ratib Anakira, ${ }^{1}$ A. K. Alomari, ${ }^{2}$ and I. Hashim ${ }^{1}$ \\ ${ }^{1}$ School of Mathematical Sciences, Universiti Kebangsaan Malaysia, 43600 Bangi, Selangor, Malaysia \\ ${ }^{2}$ Department of Mathematics, Faculty of Science, Hashemite University, Zarqa 13115, Jordan
}

Correspondence should be addressed to N. Ratib Anakira; alanaghreh_nedal@yahoo.com

Received 4 May 2013; Accepted 13 September 2013

Academic Editor: Sotiris Ntouyas

Copyright (C) 2013 N. Ratib Anakira et al. This is an open access article distributed under the Creative Commons Attribution License, which permits unrestricted use, distribution, and reproduction in any medium, provided the original work is properly cited.

\begin{abstract}
We extend for the first time the applicability of the optimal homotopy asymptotic method (OHAM) to find the algorithm of approximate analytic solution of delay differential equations (DDEs). The analytical solutions for various examples of linear and nonlinear and system of initial value problems of DDEs are obtained successfully by this method. However, this approach does not depend on small or large parameters in comparison to other perturbation methods. This method provides us with a convenient way to control the convergence of approximation series. The results which are obtained revealed that the proposed method is explicit, effective, and easy to use.
\end{abstract}

\section{Introduction}

Delay differential equation (DDE) is a form of differential equations in which derivative of the unknown function in a given time $t$ is specified in terms of the values at an earlier point in time.

DDEs have the general form

$$
\begin{aligned}
& u_{i}^{\prime}(x)=f\left(x, u_{i}(x), u_{i}\left(\xi_{j}(x)\right)\right), \\
& i=1,2, \ldots, M, \quad j=1,2, \ldots, N,
\end{aligned}
$$

where $\xi_{j}(x)=a_{j} x+b_{j}$ is the delay function.

Many problems of physics, biological models, control system, and medical and biochemical fields are modelled by DDEs. Recent studies in such diverse fields have shown that DDEs play an important role in explaining many different phenomena. Patel et al. [1] introduced an iterative scheme for the optimal control systems described by DDEs with a quadratic cost functional. In physiology, Glass and Mackey [2] applied time delays to many physiological models. Busenberg and Tang [3] created a model for cell cycle by delay equations. In recent years, DDEs are used to design models as HIV-1 therapy for fighting a virus with another virus [4].
In the last years, a great deal of attention has been devoted to study DDEs. Hence, they are solved by numerical method and approximation approach, such as Adomian decomposition method $[5,6]$, homotopy perturbation method (HPM) $[7,8]$, multiquadric approximation scheme $[9,10]$, variational iteration method (VIM) [8, 11, 12], spline methods [13], homotopy analysis method (HAM) [14], Chebyshev polynomials [15], Galerkin method [16], Legendre wavelet method [17], differential transform method [18], and Runge-Kutta method [19]. Recently, a new approach of homotopy which is called optimal homotopy asymptotic method (OHAM) was proposed and developed by Marinca et al. [20-24] for the approximate solution nonlinear problems of thin film flow of a fourth-grade fluid and for the study of the behavior of nonlinear mechanical vibration of electrical machines. In OHAM, the control and adjustment of the convergence region are provided in a convenient way. Furthermore, the OHAM has been built in convergence criteria similar to those of HAM but with greater degree of flexibility. Islam et al. [25] have applied this method successfully to nonlinear problems and have also shown its effectiveness and accuracy. Idrees et al. [26] used OHAM to study the squeezing flow between two infinite planar plates slowly approaching each other. 
The aim of this paper is to apply OHAM to get an approximate analytic solution of DDEs. The capability of this approach is tested upon several examples which offer an approximate solution in a series form that converges to exact solution in few terms. The rest of this paper is organized as follows. In Section 2, we describe the basic idea of OHAM. In Section 3, we provide the convergent theorem for this type of equations. Section 4 presents several examples to demonstrate the efficiency of the framework. The conclusion of this study is presented in Section 5 .

\section{Description of the Method}

In this section, framework of the proposed method is given and represented in the following differential equation:

$$
\begin{array}{r}
L_{i}\left(u_{i}(x)\right)+g_{i}(x)+N_{i}\left(u_{i}(x), u_{i}\left(\xi_{j}(x)\right)\right)=0, \\
i=1,2, \ldots, N, \quad j=1,2, \ldots, M,
\end{array}
$$

where $L_{i}$ are the linear operators and $N_{i}$ are the nonlinear operators contain delay function, $u_{i}(x)$ is an unknown function, $x$ denotes an independent variable, $g_{i}(x)$ is a known function, and $\xi_{j}(x)$ are the delay functions.

According to OHAM, we construct a homotopy $h_{i}\left(v_{i}(x, p), p\right): R \times[0,1] \rightarrow R$ which satisfies

$$
\begin{aligned}
(1-p) L_{i}\left[\left(v_{i}(x, p)-u_{i, 0}(x)\right]\right. \\
=H_{i}(p)\left[L_{i} v(x, p)+g_{i}(x)\right. \\
\left.\quad+N_{i}\left(v_{i}(x, p), v_{i}\left(\xi_{j}(x), p\right)\right)\right],
\end{aligned}
$$

where $x \in R, p \in[0,1]$ is an embedding parameter, $H_{i}(p)$ is a nonzero auxiliary function for $p \neq 0, H(0)=0$, and $v(x, p)$ is an unknown function. Obviously, when $p=0$ and $p=1$ it holds that $v_{i}(x, 0)=u_{i, 0}(x)$ and $v_{i}(x, 1)=v_{i}(x)$, respectively. Thus, as $p$ varies from 0 to 1 , the solution $v_{i}(x, p)$ approach from $u_{i, 0}$ to $v_{i}(x)$, where $u_{i, 0}(x)$ is the initial guess that satisfies the linear operator and the initial conditions

$$
L_{i}\left(u_{i, 0}(x)\right)+g(x)=0 .
$$

Next, we choose the auxiliary function $H_{i}(p)$ in the form

$$
H_{i}(p)=p C_{1}+p^{2} C_{2}+p^{3} C_{3}+\cdots,
$$

where $C_{1}, C_{2}, C_{3}, \ldots$ are convergence control parameters which can be determined later. $H(p)$ can be expressed in another form as reported by Herişanu and Marinca [24].

To get an approximate solution, we expand $v_{i}\left(x, p, C_{k}\right)$ in Taylor's series about $p$ in the following manner:

$$
v_{i}\left(x, p, C_{k}\right)=u_{i, 0}(x)+\sum_{k=1}^{\infty} u_{i, k}\left(x, C_{1}, C_{2}, \ldots, C_{k}\right) p^{k} .
$$

By substituting (6) into (3) and equating the coefficient of like powers of $p$, we obtain the following linear equations. Define the vectors

$$
\begin{gathered}
\vec{C}_{i}=\left\{C_{1}, C_{2}, \ldots, C_{i}\right\}, \\
\vec{u}_{i, s}=\left\{u_{i, 0}(x), u_{i, 1}\left(x, C_{1}\right), \ldots, u_{i, s}\left(x, \vec{C}_{s}\right), u_{i, 0}\left(\xi_{j}(x)\right),\right. \\
\left.u_{i, 1}\left(\xi_{j}(x), C_{1}\right), \ldots, u_{i, s}\left(\xi_{j}(x), \vec{C}_{s}\right)\right\},
\end{gathered}
$$

where $s=1,2,3, \ldots$ and $j=1,2, \ldots, M$. The zeroth-order problem is given by (4), and the first- and second-order problems are given as

$$
\begin{gathered}
L_{i}\left(u_{i, 1}(x)\right)=C_{1} N_{i, 0}\left(\vec{u}_{i, 0}\right)+g(x), \\
L_{i}\left(u_{i, 2}(x)\right)-L_{i}\left(u_{i, 1}(x)\right) \\
=C_{2} N_{i, 0}\left(\vec{u}_{i, 0}\right)+C_{1}\left[L_{i}\left(u_{i, 1}(x)\right)+N_{i, 1}\left(\vec{u}_{i, 1}\right)\right] .
\end{gathered}
$$

The general governing equations for $u_{k}(x)$ are

$$
\begin{aligned}
& L\left(u_{k}(x)\right)-L\left(u_{k-1}(x)\right) \\
& =C_{k} N_{0}\left(u_{0}(x)\right) \\
& \quad+\sum_{m=1}^{k-1} C_{m}\left[L\left(u_{k-m}(x)\right)+N_{k-m}\left(\vec{u}_{i, k-1}\right)\right],
\end{aligned}
$$

where $k=2,3, \ldots$ and $N_{i, m}\left(u_{i, 0}(x), u_{i, 1}(x), \ldots, u_{i, m}(x)\right)$ is the coefficient of $p^{m}$ in the expansion of $N(v(x, p))$ about the embedding parameter $p$ :

$$
N_{i}\left(v\left(x, p, C_{i}\right)\right)=N_{i, 0}\left(u_{0}(x)\right)+\sum_{m=1}^{\infty} N_{i, m}\left(\vec{u}_{i, m}\right) p^{m} .
$$

It has been observed that the convergence of the series (6) depends upon the auxiliary constants $C_{1}, C_{2}, C_{3}, \ldots$ If it is convergent at $p=1$, one has

$$
v_{i}\left(x, C_{k}\right)=u_{0}(x)+\sum_{k=1}^{\infty} u_{i, k}\left(x, C_{1}, C_{2}, \ldots, C_{k}\right) .
$$

The result of the $m$ th-order approximation is given as

$$
\widetilde{v}_{i}\left(x, C_{k}\right)=u_{0}(x)+\sum_{k=1}^{m} u_{i, k}\left(x, C_{1}, C_{2}, \ldots, C_{k}\right) .
$$

Substituting (12) into (2) yields the following residual:

$$
\begin{aligned}
R_{i} & \left(x, C_{1}, C_{2}, C_{3}, \ldots, C_{m}\right) \\
= & L_{i}\left(\widetilde{v}_{i}\left(x, C_{1}, C_{2}, C_{3}, \ldots, C_{m}\right)\right)+g_{i}(x) \\
& +N_{i}\left(\widetilde{v}_{i}\left(x, C_{1}, C_{2}, C_{3}, \ldots, C_{m}\right)\right) .
\end{aligned}
$$

If $R_{i}=0$, then $\tilde{u}$ will be the exact solution. Generally such a case will not arise for nonlinear problems, but we can minimize the functional

$$
J_{i}\left(C_{1}, C_{2}, C_{3}, \ldots, C_{m}\right)=\int_{a}^{b} R_{i}^{2}\left(x, C_{1}, C_{2}, C_{3}, \ldots, C_{m}\right) d x,
$$


where $a$ and $b$ are the endpoints of the given problem. The unknown convergence control parameters $C_{i}(i=$ $1,2,3, \ldots, m)$ can be calculated from the system of equations

$$
\frac{\partial J_{i}}{\partial C_{i}}=0, \quad i=1,2, \ldots, m
$$

It should be noted that our process included the auxiliary function $H_{i}(p)$ which provides us an easy way to set and optimally control the convergent area and the rate of the solution series.

\section{Convergence Theorem}

In this section, we introduce the convergence of the solution for DDEs.

Theorem 1. If the series (12) converges to $u(x)$, where $u_{k}(x) \in$ $L\left(R^{+}\right)$is produced by (8) and the $k$-order deformation (10), then $u(x)$ is the exact solution of (2).

Proof. Since the series

$$
\sum_{k=1}^{\infty} u_{i, k}\left(x, C_{1}, C_{2}, \ldots, C_{k}\right)
$$

converges, it can be written as

$$
S_{i}(x)=\sum_{k=1}^{\infty} u_{i, k}\left(x, C_{1}, C_{2}, \ldots, C_{k}\right)
$$

and it holds that

$$
\lim _{k \rightarrow \infty} u_{i, k}\left(x, C_{1}, C_{2}, \ldots, C_{k}\right)=0
$$

The left hand-side of (10) satisfies

$$
\begin{aligned}
u_{i, 1} & \left(x, C_{1}\right)+\sum_{k=2}^{n} u_{i, k}\left(x, \vec{C}_{k}\right)-\sum_{k=2}^{n} u_{i, k-1}\left(x, \vec{C}_{k-1}\right) \\
= & u_{i, 2}\left(x, \vec{C}_{2}\right)-u_{i, 1}\left(x, C_{1}\right)+\cdots+u_{i, n}\left(x, \vec{C}_{n}\right) \\
& -u_{i, n-1}\left(x, \vec{C}_{n-1}\right) \\
= & u_{i, n}\left(x, \vec{C}_{n}\right) .
\end{aligned}
$$

According to (18) we have

$$
\begin{gathered}
u_{i, 1}\left(x, C_{1}\right)+\sum_{k=2}^{n} u_{i, k}\left(x, \vec{C}_{k}\right)-\sum_{k=2}^{n} u_{i, k-1}\left(x, \vec{C}_{k-1}\right) \\
=\lim _{n \rightarrow \infty} u_{i, n}\left(x, \vec{C}_{n}\right)=0 .
\end{gathered}
$$

Using the linear operator $L_{i}$,

$$
\begin{aligned}
& L_{i}\left(u_{i, 1}\left(x, C_{1}\right)\right)+\sum_{k=2}^{\infty} L_{i}\left(u_{i, k}\left(x, \vec{C}_{k}\right)\right) \\
& -\sum_{k=2}^{\infty} L_{i}\left(u_{i, k-1}\left(x, \vec{C}_{k-1}\right)\right) \\
& =L_{i}\left(u_{i, 1}\left(x, C_{1}\right)\right)+L_{i} \sum_{k=2}^{\infty} u_{i, k}\left(x, \vec{C}_{k}\right) \\
& -L_{i} \sum_{k-1}^{\infty} u_{i, k-1}\left(x, \vec{C}_{k-1}\right)=0
\end{aligned}
$$

which satisfies

$$
\begin{gathered}
L_{i}\left(u_{i, 1}\left(x, C_{1}\right)\right)+L_{i} \sum_{k=2}^{\infty} u_{i, k}\left(x, \vec{C}_{k}\right)-L_{i} \sum_{k-1}^{\infty} u_{i, k-1}\left(x, \vec{C}_{k-1}\right) \\
=\sum_{k=2}^{\infty}\left[C_{k} N_{i, 0}\left(u_{i, 0}(x)\right)\right. \\
+\sum_{m=1}^{k-1} C_{m}\left[L_{i}\left(u_{i, k-m}\left(x, \vec{C}_{k-m}\right)\right)\right. \\
\left.\left.+N_{i, k-1-m}\left(\vec{u}_{i, k-1}\right)\right]\right]+g_{i}(x)=0 .
\end{gathered}
$$

Also the right hand side can be written as

$$
\begin{aligned}
\sum_{k=1}^{\infty}\left[\sum_{m=1}^{k} C_{m-k}\right. & \\
\times & {\left.\left[L_{i}\left(u_{i, m-1}\left(x, \vec{C}_{m-1}\right)\right)+N_{i, m-1}\left(\vec{u}_{i, k-1}\right)\right]\right] }
\end{aligned}
$$

$$
+g_{i}(x)=0 .
$$

Now, if the $C_{m}, m=1,2, \ldots$, is properly chosen, then (24) leads to

$$
L_{i}\left(u_{i}(x)\right)+N_{i}\left(u_{i}(x), u_{i}\left(\xi_{j}(x)\right)\right)+g_{i}(x)=0,
$$

which is the exact solution.

\section{Applications}

In this section, we will present a few examples with a known analytic solution in order to demonstrate the effectiveness and high precision of this algorithm.

Example 1. Consider the following linear delay differential equation [5]:

$$
u^{\prime}(x)=\frac{1}{2} e^{x / 2} u\left(\frac{x}{2}\right)+\frac{1}{2} u(x), \quad 0 \leq x \leq 1, u(0)=1,
$$


with the exact solution

$$
u(x)=e^{x} .
$$

Applying the procedure which is described in Section 2, the linear and nonlinear operators are

$$
L[v(x, p)]=\frac{d v(x, p)}{d x},
$$

$$
\begin{array}{r}
N[v(x, p)] \\
=\frac{d v(x, p)}{d x}-\frac{1}{2} \psi(x ; p) v\left(\frac{x}{2}, p\right)-\frac{1}{2} v(x, p), \\
v(0, p)=1,
\end{array}
$$

where $\psi(x ; p)$ is the expansion Taylor series of $e^{x / 2}$ with respect to $p$, which can be written as

$$
\psi(x ; p)=\sum_{k=0}^{\infty} \frac{1}{k !}\left(\frac{x}{2}\right)^{k} p^{k}
$$

Now, apply (4) to $p=0$ to give the zeroth-order problem as

$$
u_{0}^{\prime}(x)=0, \quad u_{0}(0)=1 .
$$

The solution of the zeroth-order deformation is

$$
u_{0}(x)=0 .
$$

The first-order deformation which is obtained from (8) is given as

$$
u_{1}^{\prime}\left(x, C_{1}\right)=-\frac{1}{4}(4+x) C_{1}, \quad u_{0}(0)=0,
$$

and has the solution

$$
u_{1}\left(x, C_{1}\right)=-C_{1} x-\frac{1}{8} C_{1} x^{2}
$$

The second-order deformation is given by (9):

$$
\begin{aligned}
u_{2}^{\prime}\left(x, C_{1}, C_{2}\right)= & u_{1}^{\prime}\left(x, C_{1}\right)-C_{2}-\frac{1}{4} x C_{2} \\
& -\frac{1}{16} x^{2} C_{2}-\frac{1}{2} C_{1} u_{1}\left(\frac{x}{2}, C_{1}\right) \\
& -\frac{1}{4} x C_{1} u_{1}\left(\frac{x}{2}, C_{1}\right)-\frac{1}{16} x^{2} C_{1} u_{1}\left(\frac{x}{2}, C_{1}\right) \\
& -\frac{1}{2} C_{1} u_{1}\left(x, C_{1}\right)+C_{1} u_{1}^{\prime}\left(x, C_{1}\right),
\end{aligned}
$$

with initial condition

$$
u_{2}(0)=0 .
$$

The solution of (34) is given by

$$
\begin{aligned}
u_{2}\left(x, C_{1}, C_{2}\right)=- & C_{1} x-\frac{1}{8} C_{1} x^{2}-C_{1}^{2} x+\frac{1}{4} C_{1}^{2} x^{2} \\
& +\frac{13}{192} C_{1}^{2} x^{3}+\frac{5}{512} C_{1}^{2} x^{4}+\frac{1}{2560} C_{1}^{2} x^{5} \\
& -C_{2} x-\frac{1}{8} C_{2} x^{2}-\frac{1}{48} C_{2} x^{3} .
\end{aligned}
$$

According to (10), the third-order deformation is defined as

$$
\begin{aligned}
u_{3}^{\prime}(x, & \left.C_{1}, C_{2}, C_{3}\right) \\
= & u_{2}^{\prime}\left(x, C_{1}, C_{2}\right)-C_{3}-\frac{1}{4} C_{3} x-\frac{1}{16} C_{3} x^{2}-\frac{1}{96} C_{3} x^{3} \\
& -\frac{1}{2} C_{2} u_{1}\left(\frac{x}{2}, C_{1}\right)-\frac{1}{4} C_{2} x u_{1}\left(\frac{x}{2}, C_{1}\right) \\
& -\frac{1}{16} C_{2} x^{2} u_{1}\left(\frac{x}{2}, C_{1}\right)-\frac{1}{96} C_{2} x^{3} u_{1}\left(\frac{x}{2}, C_{1}\right) \\
& -\frac{1}{2} C_{2} u_{1}\left(x, C_{1}\right)-\frac{1}{2} C_{1} u_{2}\left(\frac{x}{2}, C_{1}, C_{2}\right) \\
& -\frac{1}{4} C_{1} x u_{2}\left(\frac{x}{2}, C_{1}, C_{2}\right)-\frac{1}{16} C_{1} x^{2} u_{2}\left(\frac{x}{2}, C_{1}, C_{2}\right) \\
& -\frac{1}{96} C_{1} x^{3} u_{2}\left(\frac{x}{2}, C_{1}, C_{2}\right)-\frac{1}{2} C_{1} u_{2}\left(x, C_{1}, C_{2}\right) \\
& +C_{2} u_{1}^{\prime}\left(x, C_{1}\right)+C_{1} u_{2}^{\prime}\left(x, C_{1}, C_{2}\right),
\end{aligned}
$$

with initial condition

$$
u_{3}(0)=0,
$$

and has the solution

$$
\begin{aligned}
& u_{3}\left(x, C_{1}, C_{2}, C_{3}\right) \\
&=-C_{1} x-\frac{1}{8} C_{1} x^{2}-2 C_{1}^{2} x+\frac{1}{2} C_{1}^{2} x^{2}+\frac{13}{96} C_{1}^{2} x^{3} \\
&+\frac{5}{256} C_{1}^{2} x^{4}+\frac{7}{3840} C_{1}^{2} x^{5}+\frac{1}{18432} C_{1}^{2} x^{6} \\
&-C_{1}^{3} x+\frac{5}{8} C_{1}^{3} x^{2}+\frac{11}{192} C_{1}^{3} x^{3}+\frac{17}{4096} C_{1}^{3} x^{4} \\
&-\frac{199}{245760} C_{1}^{3} x^{5}-\frac{377}{1474560} C_{1}^{3} x^{6} \\
&-\frac{109}{5898240} C_{1}^{3} x^{7}-\frac{7}{7864320} C_{1}^{3} x^{8} \\
&-\frac{1}{707788800} C_{1}^{3} x^{9}-C_{2} x-\frac{1}{8} C_{2} x^{2}-\frac{1}{48} C_{2} x^{3} \\
&-2 C_{1} C_{2} x+\frac{1}{2} C_{1} C_{2} x^{2}+\frac{11}{96} C_{1} C_{2} x^{3} \\
&+\frac{23}{1024} C_{1} C_{2} x^{4}-\frac{23}{7680} C_{1} C_{2} x^{5}
\end{aligned}
$$




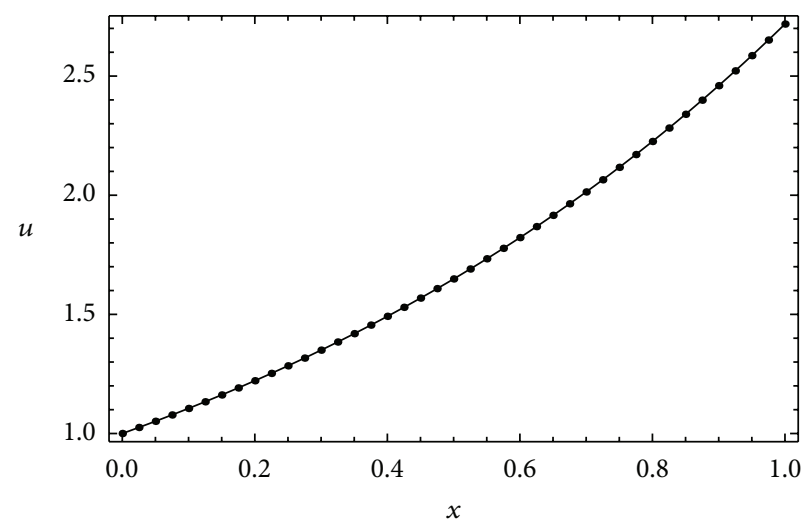

-. Exact solution

- OHAM solution

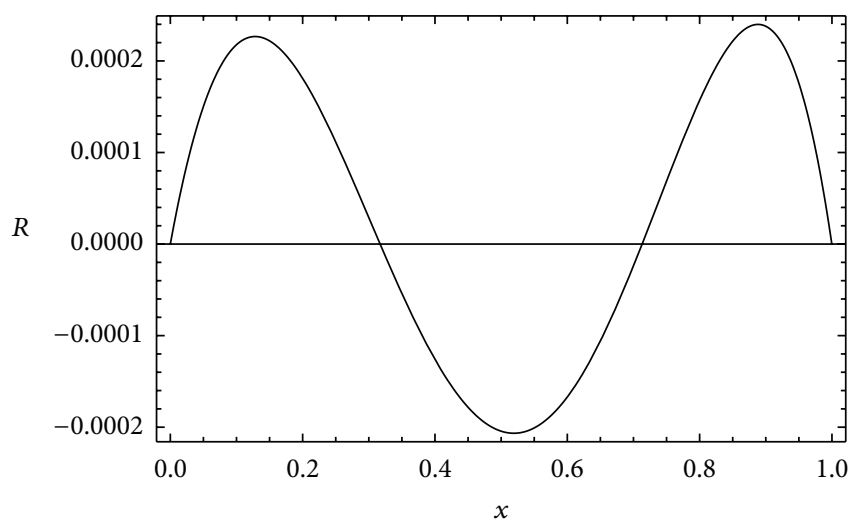

(b)

FIGURE 1: (a) Comparison between the three-term OHAM approximate solution and the exact solution for Example 1 and (b) residual error $R(x)$ given by (41) using the three-term OHAM approximate solution.

$$
\begin{aligned}
& +\frac{5}{36864} C_{1} C_{2} x^{6}+\frac{1}{258048} C_{1} C_{2} x^{7} \\
& -C_{3} x-\frac{1}{8} C_{3} x^{2}-\frac{1}{48} C_{3} x^{3}-\frac{1}{384} C_{3} x^{4}
\end{aligned}
$$

By using (31), (33), (36), and (39), the third-order approximate solution by OHAM for $p=1$ is

$$
\begin{aligned}
\tilde{u}\left(x, C_{1}, C_{2}, C_{3}\right)= & u_{0}(x)+u_{1}\left(x, C_{1}\right)+u_{2}\left(x, C_{1}, C_{2}\right) \\
& +u_{3}\left(x, C_{1}, C_{2}, C_{3}\right) .
\end{aligned}
$$

By using the proposed method of Section 2 on $[0,1]$, we use the residual error:

$$
\begin{aligned}
R= & \tilde{u}^{\prime}\left(x, C_{1}, C_{2}, C_{3}\right)-\frac{1}{2} e^{x / 2} \tilde{u}\left(\frac{x}{2}, C_{1}, C_{2}, C_{3}\right) \\
& -\frac{1}{2} \widetilde{u}\left(x, C_{1}, C_{2}, C_{3}\right) .
\end{aligned}
$$

The Less Square error can be formed as

$$
\begin{gathered}
J\left(C_{1}, C_{2}, C_{3}\right)=\int_{0}^{1} R^{2} d x \\
\frac{\partial J\left(C_{1}, C_{2}, C_{3}\right)}{\partial C_{1}}=\frac{\partial J\left(C_{1}, C_{2}, C_{3}\right)}{\partial C_{2}}=\frac{\partial J\left(C_{1}, C_{2}, C_{3}\right)}{\partial C_{3}}=0 .
\end{gathered}
$$

Thus, the following optimal values of $C_{i}$ 's are obtained:

$$
\begin{gathered}
C_{1}=-1.1862449850, \quad C_{2}=0.0255300261, \\
C_{3}=-0.0070171914 .
\end{gathered}
$$

In this case, our approximate solution is

$$
\begin{aligned}
\tilde{u}(x, & \left.C_{1}, C_{2}, C_{3}\right) \\
= & 1+1.00397 x+0.479341 x^{2}+0.194878 x^{3} \\
& +0.0349963 x^{4}+0.00455719 x^{5} \\
& +0.00050723 x^{6}+0.0000309654 x^{7} \\
& +1.4858 \times 10^{-6} x^{8}+2.35841 \times 10^{-8} x^{9}
\end{aligned}
$$

Equations (44) and (41) are plotted in Figures 1(a) and 1(b), respectively. Figure 1(a) shows a comparison between the approximate solution which is obtained by using OHAM and exact solution (27). The residual error is plotted in Figure 1(b). We noted that the absolute maximum error for solving this example via HAM is 0.04 while the absolute maximum error via OHAM is $0.2 \times 10^{-3}$, which leads to conclude that OHAM is more accurate than HAM.

Example 2. Consider the linear delay differential equation of third order [5]

$$
\begin{gathered}
u^{\prime \prime \prime}(x)=-u(x)-u(x-0.3)+e^{-x+0.3}, \quad 0 \leq x \leq 1, \\
u^{\prime}(0)=-1, \quad u^{\prime \prime}(0)=1, \quad u(x)=e^{-x}, \quad x \leq 0,
\end{gathered}
$$

with exact solution

$$
u(x)=e^{-x}
$$


According to the method which was described in the above section, we start with

$$
\begin{gathered}
L[v(x, p)]=\frac{d^{3} v(x, p)}{d x^{3}}, \\
N[v(x, p)] \\
=\frac{d^{3} v(x, p)}{d x^{3}}+v(x, p)+v(x-0.3, p)-e^{-x+0.3} .
\end{gathered}
$$

By applying OHAM, we have the following zero-, first-, second-, and the third-order approximate solutions:

$$
\begin{aligned}
& u_{0}(x)=1-x+0.5 x^{2} \\
& u_{1}\left(x, C_{1}\right)=0.174167 C_{1} x^{3} \\
& -0.0541667 C_{1} x^{4}+0.0166667 C_{1} x^{5}, \\
& u_{2}\left(x, C_{1}, C_{2}\right) \\
& =0.174167 C_{1} x^{3}-0.0541667 C_{1} x^{4} \\
& +0.0166667 C_{1} x^{5}+0.173303 C_{1}^{2} x^{3} \\
& -0.0519354 C_{1}^{2} x^{4}+0.0134917 C_{1}^{2} x^{5} \\
& +0.00356944 C_{1}^{2} x^{6}-0.000634921 C_{1}^{2} x^{7} \\
& +0.0000992063 C_{1}^{2} x^{8}+0.166667 C_{2} x^{3} \\
& -0.0416667 C_{2} x^{4}+0.00833333 C_{2} x^{5}, \\
& u_{3}\left(x, C_{1}, C_{2}, C_{3}\right) \\
& =0.174167 C_{1} x^{3}-0.0541667 C_{1} x^{4} \\
& +0.0166667 C_{1} x^{5}+0.346606 C_{1}^{2} x^{3} \\
& -0.103871 C_{1}^{2} x^{4}+0.0269833 C_{1}^{2} x^{5} \\
& +0.00713889 C_{1}^{2} x^{6}-0.00126984 C_{1}^{2} x^{7} \\
& +0.000198413 C_{1}^{2} x^{8}+0.172448 C_{1}^{3} x^{3} \\
& -0.0497316 C_{1}^{3} x^{4}+0.0103718 C_{1}^{3} x^{5} \\
& +0.0070607 C_{1}^{3} x^{6}-0.00119984 C_{1}^{3} x^{7} \\
& +0.000156374 C_{1}^{3} x^{8}+0.000017306 C_{1}^{3} x^{9} \\
& -2.09436 \times 10^{-6} C_{1}^{3} x^{10} \\
& +2.00417 \times 10^{-7} C_{1}^{3} x^{11}+0.166667 C_{2} x^{3} \\
& -0.0416667 C_{2} x^{4}+0.00833333 C_{2} x^{5} \\
& +0.33916 C_{1} C_{2} x^{3}-0.0915255 C_{1} C_{2} x^{4} \\
& +0.0189125 C_{1} C_{2} x^{5}+0.00682639 C_{1} C_{2} x^{6}
\end{aligned}
$$

$$
\begin{aligned}
& \text { By substituting values in (52), we have } \\
& \begin{aligned}
\widetilde{u}\left(x, C_{1}, C_{2}, C_{3}\right) \\
=1-x+0.5 x^{2}-0.161547 x^{3}+0.0319249 x^{4} \\
\quad-0.00106469 x^{5}-0.000894311 x^{6}+0.0000269763 x^{7} \\
\quad-1.86401 \times 10^{-6} x^{8}+1.93748 \times 10^{-7} x^{9} \\
\quad-2.34472 \times 10^{-8} x^{10}+2.24376 \times 10^{-9} x^{11} .
\end{aligned}
\end{aligned}
$$

The comparison between the approximate solution and the exact solution is shown in Figures 2(a) and 2(b). We observe that the results agree very well with the exact solution.

Example 3. Consider the first order of nonlinear delay differential equation [14]

$$
u^{\prime}(x)=-2 u^{2}\left(\frac{x}{2}\right)+1, \quad 0 \leq x \leq 1, u(0)=0,
$$

which has the exact solution

$$
u(x)=\sin (x)
$$

By applying the same method as in Examples 1 and 2, we have the following:

$$
\begin{gathered}
L[v(x, p)]=\frac{d v(x, p)}{d x}, \\
N[v(x, p)]=\frac{d v(x, p)}{d x}+2 v^{2}\left(\frac{x}{2}, p\right)-1 .
\end{gathered}
$$




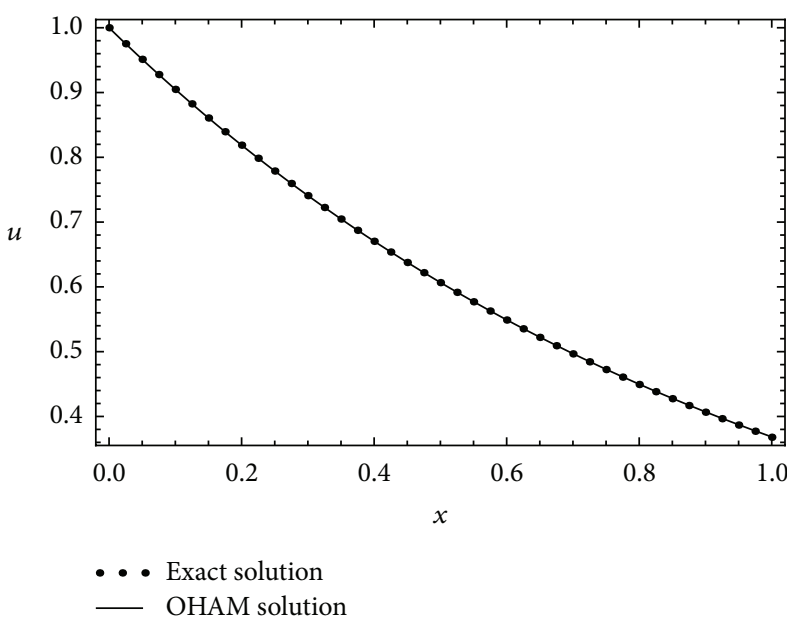

(a)

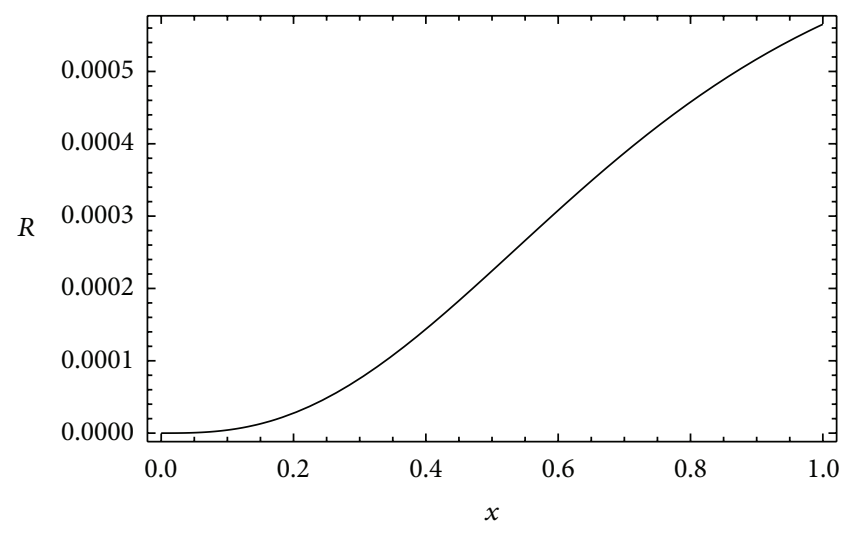

(b)

FIgURE 2: (a) Comparison between the three-term OHAM approximate solution and the exact solution for Example 2 and (b) residual error $R(x)$ given by (50) using three-term OHAM approximate solution.

According to OHAM, we have the following zero-, first-, second- and the third-order approximate solutions:

$$
\begin{gathered}
u_{0}(x)=x, \\
u_{1}\left(x, C_{1}\right)=\frac{1}{6} x^{3}, \\
u_{2}\left(x, C_{1}, C_{2}\right)=\frac{1}{6} C_{1} x^{3}+\frac{1}{6} C_{1}^{2} x^{3}+\frac{1}{120} C_{1}^{2} x^{5}+\frac{1}{6} C_{2} x^{3}, \\
u_{3}\left(x, C_{1}, C_{2}, C_{3}\right) \\
=\frac{1}{6} C_{1} x^{3}+\frac{1}{3} C_{1}^{2} x^{3}+\frac{1}{60} C_{1}^{2} x^{5}+\frac{1}{6} C_{1}^{3} x^{3} \\
+\frac{1}{60} C_{1}^{3} x^{5}+\frac{1}{5040} C_{1}^{3} x^{7}+\frac{1}{6} C_{2} x^{3}+\frac{1}{3} C_{1} C_{2} x^{3} \\
+\frac{1}{60} C_{1} C_{2} x^{5}+\frac{1}{6} C_{3} x^{3} .
\end{gathered}
$$

From (59), the third-order approximate solution by OHAM is given as

$$
\begin{aligned}
\widetilde{u}\left(x, C_{1}, C_{2}, C_{3}\right)= & u_{0}(x)+u_{1}\left(x, C_{1}\right) \\
& +u_{2}\left(x, C_{1}, C_{2}\right)+u_{3}\left(x, C_{1}, C_{2}, C_{3}\right) .
\end{aligned}
$$

By using (60) in (14) and applying the method as discussed in (15) and (16), we obtain the following values of $C_{i}$ 's:

$$
\begin{gathered}
C_{1}=-0.9892887781, \quad C_{2}=0.0001159690, \\
C_{3}=3.3939542758 .
\end{gathered}
$$

The approximate solution now becomes

$$
\begin{aligned}
\widetilde{u}\left(x, C_{1}, C_{2}, C_{3}\right)= & x-0.166665 x^{3} \\
& +0.00832857 x^{5}-0.000192105 x^{7} .
\end{aligned}
$$

From Figures 3(a) and 3(b), we observe that the results agree very well with the exact solution; as we increase the order of the problem the accuracy increases and the residual error will decrease as shown in Figure 3(b). We observed that the absolute maximum error for solving this example via HAM is $1.2 \times 10^{-6}$ while the absolute maximum error by using OHAM is $4 \times 10^{-8}$, which revealed that the proposed method is more accurate than HAM.

Example 4. Consider the third-order nonlinear delay differential equation [14]

$$
\begin{gathered}
u^{\prime \prime \prime}(x)=2 u^{2}\left(\frac{x}{2}\right)-1, \\
0 \leq x \leq 1, u(0)=0, u^{\prime}(0)=1, u^{\prime \prime}(0)=0 .
\end{gathered}
$$

The exact solution of the above problem is given as

$$
u(x)=\sin (x)
$$

By applying the present method, the linear and nonlinear operators are defined as

$$
\begin{gathered}
L[v(x, p)]=\frac{d^{3} v(x, p)}{d x^{3}}, \\
N[v(x, p)]=\frac{d^{3} v(x, p)}{d x^{3}}-2 v^{2}\left(\frac{x}{2}, p\right)+1 .
\end{gathered}
$$




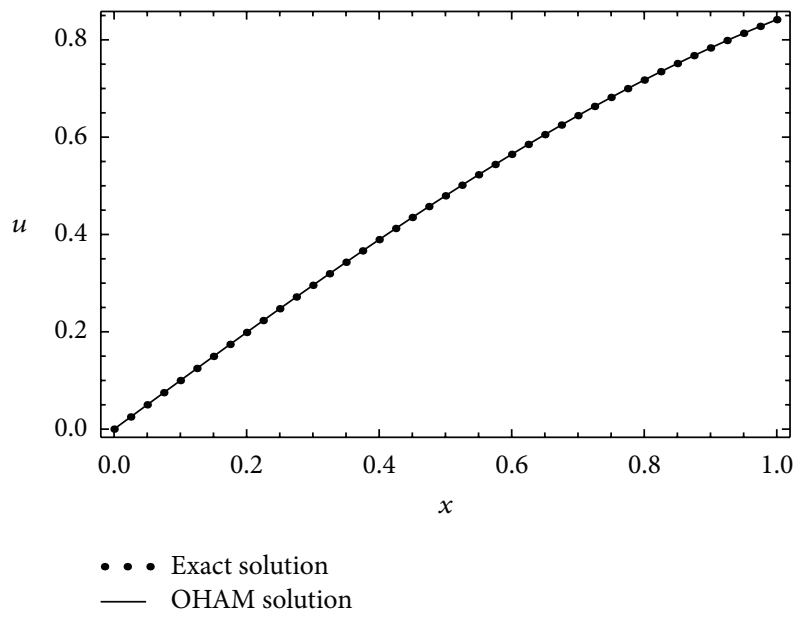

(a)

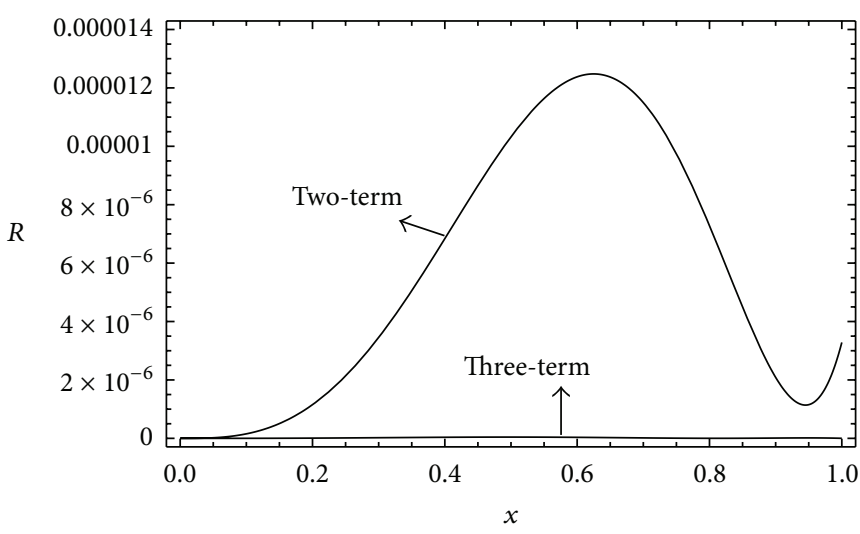

(b)

FIGURE 3: (a) Comparison between three-term OHAM approximate solution and the exact solution for Example 3 and (b) the residual error for Example 3 using two-term and three-term OHAM approximate solutions.

According to OHAM, we have the following zero-, first-, second- and third-order approximate solutions:

$$
\begin{aligned}
& u_{0}(x)=x-\frac{x^{3}}{6} \\
& u_{1}\left(x, C_{1}\right)=-\frac{1}{120} C_{1} x^{5}+\frac{1}{5040} C_{1} x^{7}-\frac{1}{580608} C_{1} x^{9} \\
& u_{2}\left(x, C_{1}, C_{2}\right) \\
& =-\frac{1}{120} C_{1} x^{5}+\frac{1}{5040} C_{1} x^{7}-\frac{1}{580608} C_{1} x^{9} \\
& -\frac{1}{120} C_{1}^{2} x^{5}+\frac{1}{5040} C_{1}^{2} x^{7}-\frac{1}{1451520} C_{1}^{2} x^{9} \\
& -\frac{1}{39916800} C_{1}^{2} x^{11}+\frac{101}{1275293859840} C_{1}^{2} x^{13} \\
& -\frac{1}{9738607656960} C_{1}^{2} x^{15}-\frac{1}{120} C_{2} x^{5} \\
& +\frac{1}{5040} C_{2} x^{7}-\frac{1}{580608} C_{2} x^{9}, \\
& u_{3}\left(x, C_{1}, C_{2}, C_{3}\right) \\
& =-\frac{1}{120} C_{1} x^{5}+\frac{1}{5040} C_{1} x^{7}-\frac{1}{580608} C_{1} x^{9} \\
& -\frac{1}{60} C_{1}^{2} x^{5}+\frac{1}{2520} C_{1}^{2} x^{7}-\frac{1}{725760} C_{1}^{2} x^{9} \\
& -\frac{1}{19958400} C_{1}^{2} x^{11}+\frac{101}{637646929920} C_{1}^{2} x^{13} \\
& -\frac{1}{4869303829480} C_{1}^{2} x^{15}-\frac{1}{120} C_{3}^{3} x^{5} \\
& +\frac{1}{5040} C_{1}^{3} x^{7}+\frac{1}{2903040} C_{1}^{3} x^{9}
\end{aligned}
$$

$$
\begin{aligned}
& -\frac{1}{19958400} C_{1}^{3} x^{11}+\frac{491}{6376469299200} C_{1}^{3} x^{13} \\
& +\frac{1223}{2678117105664000} C_{1}^{3} x^{15} \\
& -\frac{341827}{149186120241276518400} C_{1}^{3} x^{17} \\
& +\frac{1}{5040} C_{3} x^{7}-\frac{1}{580608} C_{3} x^{9} \\
& -\frac{263}{91675269781634049638400} C_{1}^{3} x^{21} \\
& -\frac{1}{120} C_{2} x^{5}+\frac{1}{5040} C_{2} x^{7}-\frac{1}{580608} C_{2} x^{9} \\
& -\frac{1}{60} C_{1} C_{2} x^{5}+\frac{1}{2520} C_{1} C_{2} x^{7} \\
& -\frac{1}{725760} C_{1} C_{2} x^{9}-\frac{1}{19958400} C_{1} C_{2} x^{11} \\
& +\frac{101}{637646929920} C_{1} C_{2} x^{13}-\frac{1}{120} C_{3} x^{5} \\
& -\frac{1}{4869303828480} C_{1} C_{2} x^{15} \\
& +\frac{38033}{10204330624503313858560} C_{1}^{3} x^{19}
\end{aligned}
$$

From (66), the third-order approximate solution by OHAM is given as

$$
\begin{aligned}
\tilde{u}\left(x, C_{1}, C_{2}, C_{3}\right)= & u_{0}(x)+u_{1}\left(x, C_{1}\right) \\
& +u_{2}\left(x, C_{1}, C_{2}\right)+u_{3}\left(x, C_{1}, C_{2}, C_{3}\right) .
\end{aligned}
$$




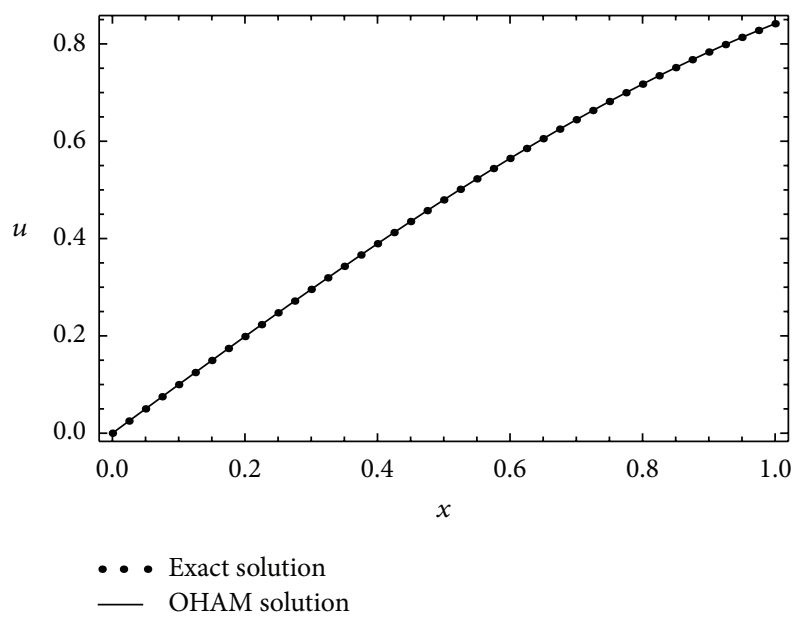

(a)

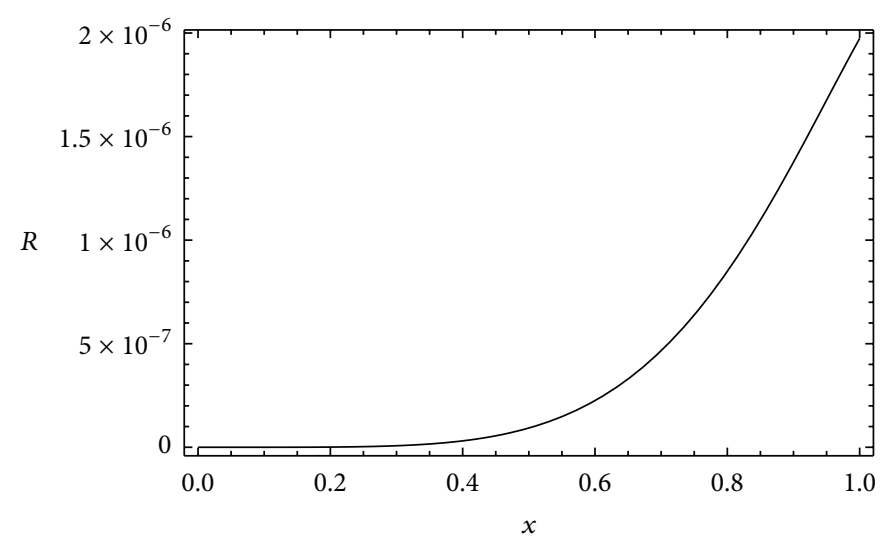

(b)

FIGURE 4: (a) Comparison between three-term OHAM approximate solution and the exact solution for Example 4 and (b) the residual error for Example 4 using three-term OHAM approximate solution.

By using (67) in (14) and applying the method as discussed in (15) and (16), we obtain the following values of $C_{i}$ 's:

$$
\begin{gathered}
C_{1}=2.7354549148, \quad C_{2}=-1.3589185618, \\
C_{3}=-1.0003665216 .
\end{gathered}
$$

The approximate solution now becomes

$$
\begin{aligned}
& \tilde{u}\left(x, C_{1}, C_{2}, C_{3}\right) \\
&=x-\frac{1}{6} x^{3}+0.00833639 x^{5}-0.000198485 x^{7} \\
&+1.72296 \times 10^{-6} x^{9}+1.80627 \times 10^{-29} x^{11} \\
&-5.71017 \times 10^{-32} x^{13}+7.40356 \times 10^{-35} x^{15} \\
&-4.68993 \times-50 x^{17}+7.62895 \times 10^{53} x^{19} \\
&-5.87208 \times 10^{-56} x^{21} .
\end{aligned}
$$

Numerical results of the solution are displayed in Figures 4(a) and 4(b).

Example 5. Consider the system of delay differential equation [14]

$$
\begin{aligned}
& u_{1}^{\prime}(x)=u_{1}(x-1), \\
& u_{2}^{\prime}(x)=u_{1}(x-1)+u_{2}(x-0.2), \\
& u_{3}^{\prime}(x)=u_{2}(x-1),
\end{aligned}
$$

with initial conditions

$$
u_{1}(0)=1, \quad u_{2}(0)=1, \quad u_{3}(0)=1 .
$$

Following the same procedure, we have

$$
\begin{gathered}
L_{i}\left[v_{i}(x, p)\right]=\frac{d v_{i}(x, p)}{d x}, \quad i=1,2,3, \\
N_{1}\left[v_{1}(x, p)\right]=\frac{d v_{1}(x, p)}{d x}-v_{1}(x-1, p), \\
N_{2}\left[v_{2}(x, p)\right]=\frac{d v_{2}(x, p)}{d x}-v_{1}(x-1, p)-v_{2}(x-0.2, p), \\
N_{3}\left[v_{3}(x, p)\right]=\frac{d v_{3}(x, p)}{d x}-v_{2}(x-1, p) .
\end{gathered}
$$

According to OHAM formulation, we have the following: zeroth-order solution:

$$
\begin{aligned}
& u_{1,0}(x)=1, \\
& u_{2,0}(x)=1, \\
& u_{3,0}(x)=1,
\end{aligned}
$$

first-order solution:

$$
\begin{aligned}
& u_{1,1}(x)=-C_{1} x, \\
& u_{2,1}(x)=-2 K_{1} x, \\
& u_{3,1}(x)=-A_{1} x,
\end{aligned}
$$

second-order solution:

$$
\begin{gathered}
u_{1,2}(x)=-C_{1} x-2 C_{1}^{2} x+\frac{1}{2} C_{1}^{2} x^{2}-C_{2} x, \\
u_{2,2}(x)=-2 K_{1} x-3.4 K_{1}^{2} x+\frac{3}{2} K_{1}^{2} x^{2}-2 K_{2} x, \\
u_{3,2}(x)=-A_{1} x-3 A_{1}^{2} x+A_{1}^{2} x^{2}-A_{2} x .
\end{gathered}
$$


Making use of (73)-(75) and extending the solutions up to a fifth order, the approximate solutions by OHAM for $p=1$ are

$$
\begin{aligned}
\tilde{u}_{1}\left(x, C_{1}, C_{2}, C_{3}, C_{4}, C_{5}\right) \\
=u_{1,0}(x)+u_{1,1}\left(x, C_{1}\right)+u_{1,2}\left(x, C_{1}, C_{2}\right) \\
\quad+u_{1,3}\left(x, C_{1}, C_{2}, C_{3}\right)+u_{1,4}\left(x, C_{1}, C_{2}, C_{3}, C_{4}\right) \\
\quad+u_{1,5}\left(x, C_{1}, C_{2}, C_{3}, C_{4}, C_{5}\right) \\
\tilde{u}_{2}\left(x, K_{1}, K_{2}, K_{3}, K_{4}, K_{5}\right) \\
=u_{2,0}(x)+u_{2,1}\left(x, K_{1}\right)+u_{2,2}\left(x, K_{1}, K_{2}\right) \\
\quad+u_{2,3}\left(x, K_{1}, K_{2}, K_{3}\right)+u_{2,4}\left(x, K_{1}, K_{2}, K_{3}, K_{4}\right) \\
\quad+u_{2,5}\left(x, K_{1}, K_{2}, K_{3}, K_{4}, K_{5}\right), \\
\tilde{u}_{3}\left(x, A_{1}, A_{2}, A_{3}, A_{4}, A_{5}\right) \\
=u_{2,0}(x)+u_{2,1}\left(x, A_{1}\right)+u_{2,2}\left(x, A_{1}, A_{2}\right) \\
\quad+u_{2,3}\left(x, A_{1}, A_{2}, A_{3}\right)+u_{2,4}\left(x, A_{1}, A_{2}, A_{3}, A_{4}\right) \\
\quad+u_{2,5}\left(x, A_{1}, A_{2}, A_{3}, A_{4}, A_{5}\right) .
\end{aligned}
$$

By using the proposed procedure which is described in Section 2 on $[0,1]$, we use the residual error

$$
\begin{aligned}
R_{1}= & \widetilde{u}_{1}^{\prime}\left(x, C_{1}, C_{2}, C_{3}, C_{4}, C_{5}\right) \\
& -u_{1}\left(x-1, C_{1}, C_{2}, C_{3}, C_{4}, C_{5}\right), \\
R_{2}= & \widetilde{u}_{2}^{\prime}\left(x, K_{1}, K_{2}, K_{3}, K_{4}, K_{5}\right) \\
& -u_{1}\left(x-1, C_{1}, C_{2}, C_{3}, C_{4}, C_{5}\right) \\
& -u_{2}\left(x-0.2, K_{1}, K_{2}, K_{3}, K_{4}, K_{5}\right), \\
R_{3}= & \widetilde{u}_{3}^{\prime}\left(x, A_{1}, A_{2}, A_{3}, A_{4}, A_{5}\right) \\
& -u_{2}\left(x-1, K_{1}, K_{2}, K_{3}, K_{4}, K_{5}\right) .
\end{aligned}
$$

The following values of $C_{i}$ 's, $K_{i}$ 's and $A_{i}$ 's are obtained:

$$
\begin{array}{r}
C_{1}=-0.58668069, \quad C_{2}=-0.08423352, \\
C_{3}=0.00786513, \quad C_{4}=0.00069702, \\
C_{5}=-0.00034937, \\
K_{1}=-0.75856373, \quad K_{2}=-0.14305522, \\
K_{3}=0.07154350, \quad K_{4}=-0.02323472, \\
K_{5}=-0.00280683, \\
A_{1}=-0.67739965, \quad A_{2}=-0.08965635, \\
A_{3}=0.03646196, \quad A_{4}=-0.01330410, \\
A_{5}=0.00453433 .
\end{array}
$$

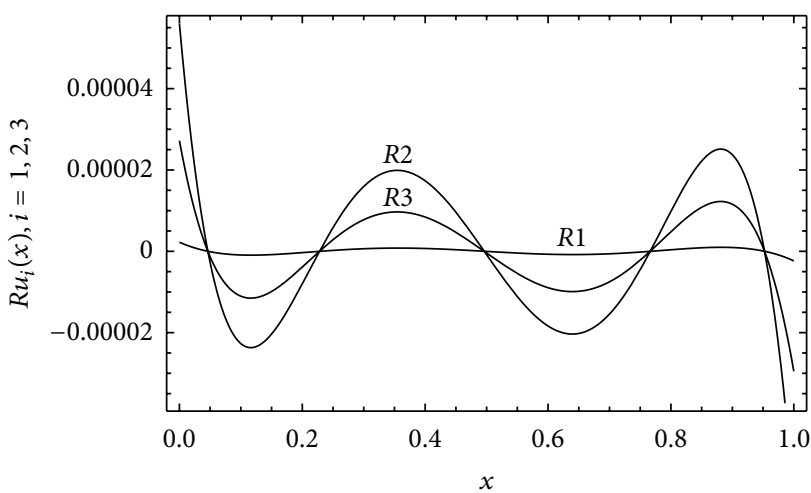

Figure 5: The residual errors for Example 5 using the five-term OHAM approximate solution.

By using the above values, the approximate solutions are

$$
\begin{aligned}
\tilde{u}_{1}\left(x, C_{1}, C_{2}, C_{3}, C_{4}, C_{5}\right) \\
=1+0.567178 x+0.160857 x^{2}+0.0302719 x^{3} \\
\quad+0.00434813 x^{4}+0.000579199 x^{5}, \\
\tilde{u}_{2}\left(x, K_{1}, K_{2}, K_{3}, K_{4}, K_{5}\right) \\
=1+1.3299 x+0.714785 x^{2}+0.205164 x^{3} \\
\quad+0.0536786 x^{4}+0.0125583 x^{5}, \\
\widetilde{u}_{2}\left(x, A_{1}, A_{2}, A_{3}, A_{4}, A_{5}\right) \\
=1+0.182376 x+0.230372 x^{2}+0.0785302 x^{3} \\
\quad+0.0228859 x^{4}+0.00594311 x^{5} .
\end{aligned}
$$

From Figure 5, we can observe the accuracy of the solution obtained by the five-term approximate solution using OHAM which is quite good.

\section{Conclusions}

In this work, OHAM is employed for the first time to propose a new analytic approximate solution of delay differential equations (DDEs). This method has been tested in various examples of linear and nonlinear and system of initial value problems of DDEs and was seen to yield satisfactory results. The OHAM provides us with a simple way to optimally control and adjust the convergence solution series and it gives a good approximation in few terms which is converged to the exact solution and proved the efficiency and reliability of the method. This fact is obvious from the use of the auxiliary function $H(p)$. In OHAM, it is important to solve a set of nonlinear algebraic equations with $m$ unknown convergence control parameters, $C_{1}, C_{2}, \ldots, C_{m}$, and this makes it time consuming, especially for large $m$. 


\section{References}

[1] N. K. Patel, P. C. Das, and S. S. Parbhu, "Optimal control of systems described by delay differential equations," International Journal of Control, vol. 36, no. 2, pp. 303-311, 1982.

[2] L. Glass and M. C. Mackey, "Pathological conditions resulting from instabilities in physiological control systems," Annals of the New York Academy of Sciences, vol. 316, pp. 214-235, 1979.

[3] S. Busenberg and B. Tang, "Mathematical models of the early embryonic cell cycle: the role of MPF activation and cyclin degradation," Journal of Mathematical Biology, vol. 32, no. 6, pp. 573-596, 1994.

[4] C. Lv and Z. Yuan, "Stability analysis of delay differential equation models of HIV-1 therapy for fighting a virus with another virus," Journal of Mathematical Analysis and Applications, vol. 352, no. 2, pp. 672-683, 2009.

[5] D. J. Evans and K. R. Raslan, "The Adomian decomposition method for solving delay differential equation," International Journal of Computer Mathematics, vol. 82, no. 1, pp. 49-54, 2005.

[6] R. K. Saeed and B. M. Rahman, "Adomian decomposition method for solving system of delay differential equation," Australian Journal of Basic and Applied Sciences, vol. 4, no. 8, pp. 3613-3621, 2010.

[7] F. Shakeri and M. Dehghan, "Solution of delay differential equations via a homotopy perturbation method," Mathematical and Computer Modelling, vol. 48, no. 3-4, pp. 486-498, 2008.

[8] H. Koçak and A. Yildirim, "Series solution for a delay differential equation arising in electrodynamics," Communications in Numerical Methods in Engineering, vol. 25, no. 11, pp. 1084-1096, 2009.

[9] S. K. Vanani and A. Aminataei, "Multiquadric approximation scheme on the numerical solution of delay differential systems of neutral type," Mathematical and Computer Modelling, vol. 49, no. 1-2, pp. 234-241, 2009.

[10] S. K. Vanani and A. Aminataei, "On the numerical solution of neutral delay differential equations using multiquadric approximation scheme," Bulletin of the Korean Mathematical Society, vol. 45, no. 4, pp. 663-670, 2008.

[11] A. Saadatmandi and M. Dehghan, "Variational iteration method for solving a generalized pantograph equation," Computers and Mathematics with Applications, vol. 58, no. 11-12, pp. 2190-2196, 2009.

[12] Y. M. Rangkuti and M. S. M. Noorani, "The exact solution of delay differential equations using coupling variational iteration with Taylor series and small term," Bulletin of Mathemaatics, vol. 4, no. 1, pp. 1-15, 2012.

[13] A. El-Safty, M. S. Salim, and M. A. El-Khatib, "Convergence of the spline function for delay dynamic system," International Journal of Computer Mathematics, vol. 80, no. 4, pp. 509-518, 2003.

[14] A. K. Alomari, M. S. M. Noorani, and R. Nazar, "Solution of delay differential equation by means of homotopy analysis method," Acta Applicandae Mathematicae, vol. 108, no. 2, pp. 395-412, 2009.

[15] S. Sedaghat, Y. Ordokhani, and M. Dehghan, "Numerical solution of the delay differential equations of pantograph type via Chebyshev polynomials," Communications in Nonlinear Science and Numerical Simulation, vol. 17, no. 12, pp. 4815-4830, 2012.

[16] K. Nandakumar and M. Wiercigroch, "Galerkin projections for state-dependent delay differential equations with applications to drilling," Applied Mathematical Modelling, vol. 37, no. 4, pp. 1705-1722, 2013.
[17] M. S. Hafshejani, S. K. Vanani, and J. S. Hafshejani, "Numerical solution of delay differential equations using Legender wavelet method," World Applied Sciences Journal, vol. 13, pp. 27-33, 2011.

[18] F. Karakoc and H. Bereketolu, "Solutions of delay differential equations by using differential transform method," International Journal of Computer Mathematics, vol. 86, no. 5, pp. 914923, 2009.

[19] D. Li and M. Z. Liu, "Runge-Kutta methods for the multipantograph delay equation," Applied Mathematics and Computation, vol. 163, no. 1, pp. 383-395, 2005.

[20] V. Marinca, N. Herişanu, C. Bota, and B. Marinca, "An optimal homotopy asymptotic method applied to the steady flow of a fourth-grade fluid past a porous plate," Applied Mathematics Letters, vol. 22, no. 2, pp. 245-251, 2009.

[21] V. Marinca, N. Herişanu, and I. Nemeş, "Optimal homotopy asymptotic method with application to thin film flow," Central European Journal of Physics, vol. 6, no. 3, pp. 648-653, 2008.

[22] V. Marinca and N. Herişanu, "Application of optimal homotopy asymptotic method for solving nonlinear equations arising in heat transfer," International Communications in Heat and Mass Transfer, vol. 35, no. 6, pp. 710-715, 2008.

[23] N. Herisanu, V. Marinca, T. Dordea, and G. Madescu, "A new analytical approach to nonlinear vibration of an electric machine," Proceedings of Romanian Academy A, vol. 9, no. 3, 2008.

[24] N. Herişanu and V. Marinca, "Explicit analytical approximation to large-amplitude non-linear oscillations of a uniform cantilever beam carrying an intermediate lumped mass and rotary inertia," Meccanica, vol. 45, no. 6, pp. 847-855, 2010.

[25] S. Islam, R. Ali Shah, I. Ali, and N. M. Allah, "Optimal homotopy asymptotic solutions of couette and poiseuille flows of a third grade fluid with heat transfer analysis," International Journal of Nonlinear Sciences and Numerical Simulation, vol. 11, no. 6, pp. 389-400, 2010.

[26] M. Idrees, S. Islam, S. Haq, and S. Islam, "Application of the optimal homotopy asymptotic method to squeezing flow," Computers and Mathematics with Applications, vol. 59, no. 12, pp. 3858-3866, 2010. 


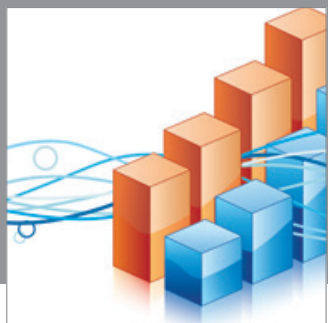

Advances in

Operations Research

mansans

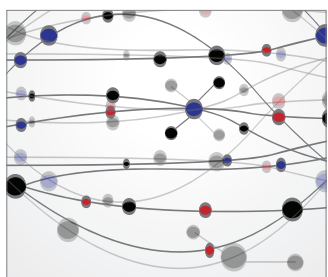

The Scientific World Journal
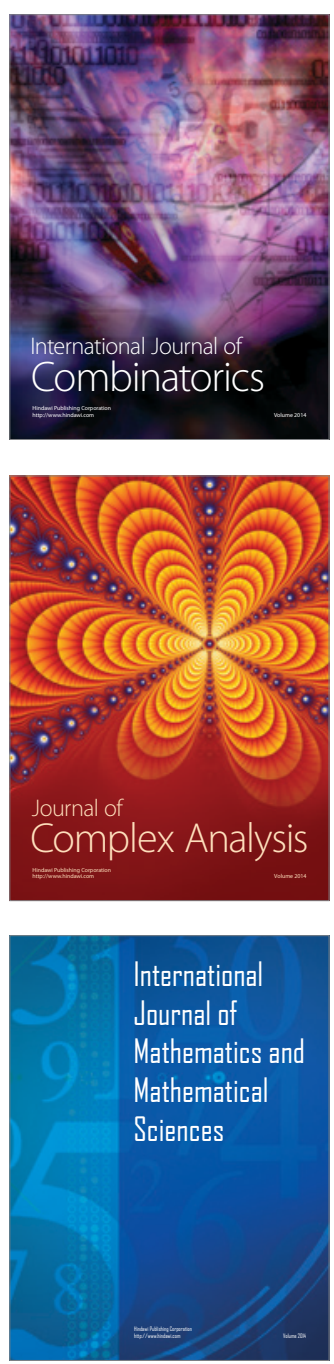
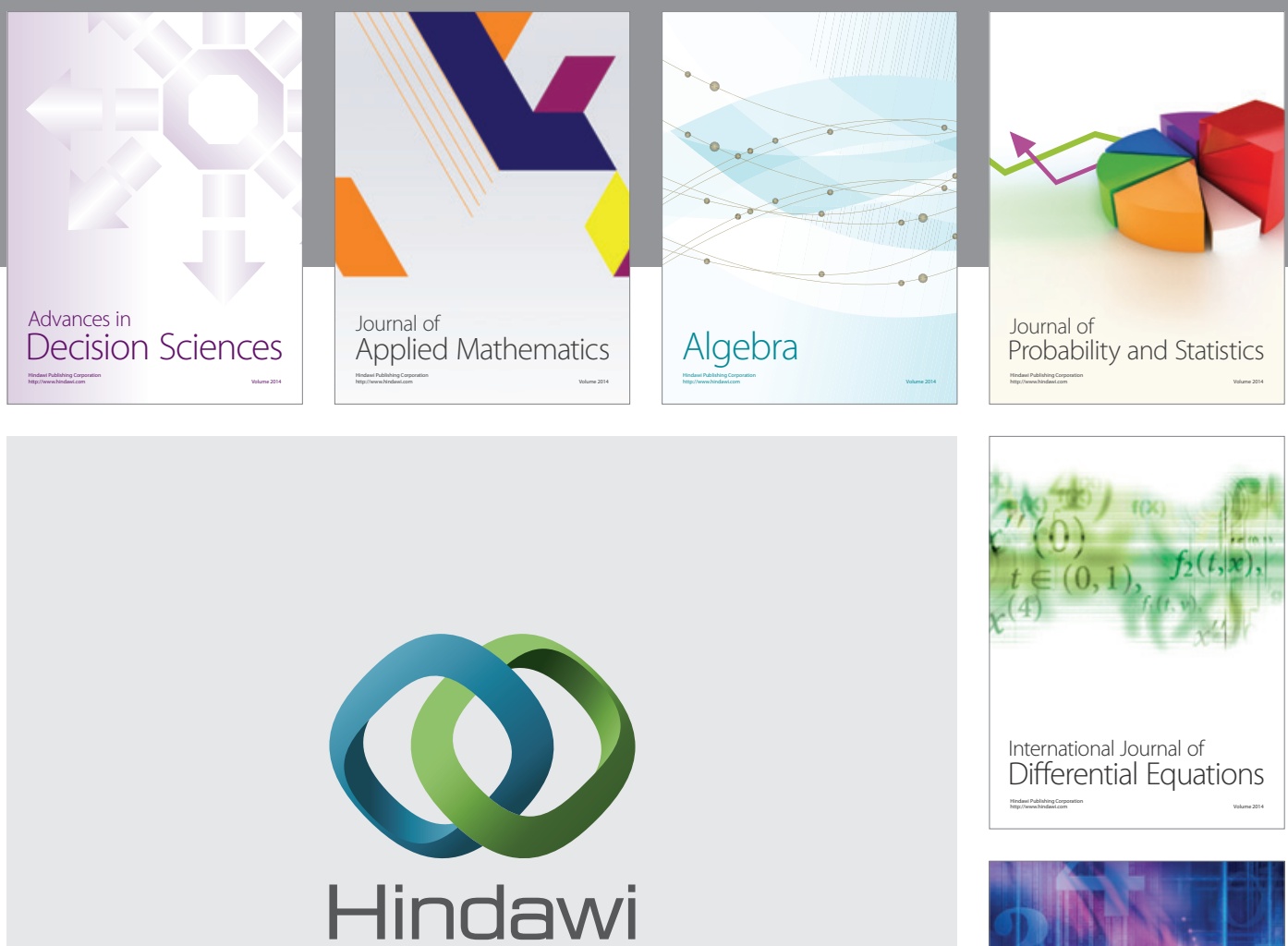

Submit your manuscripts at http://www.hindawi.com
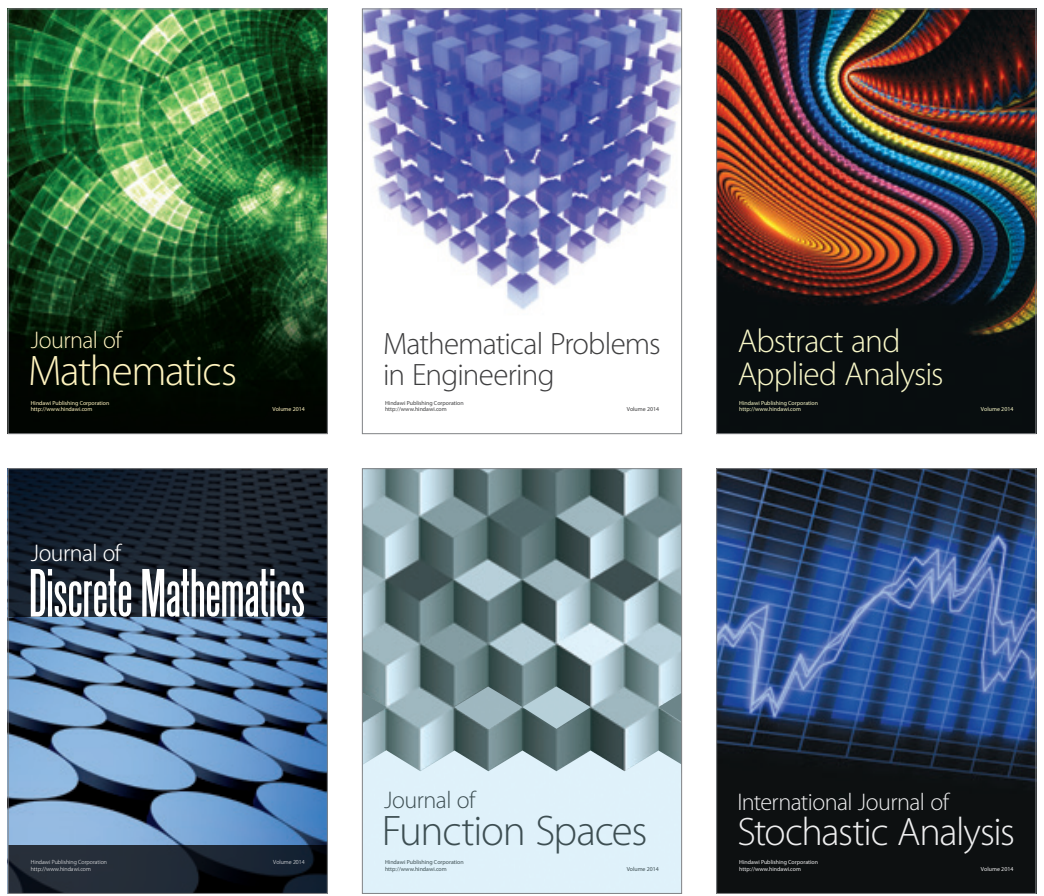

Journal of

Function Spaces

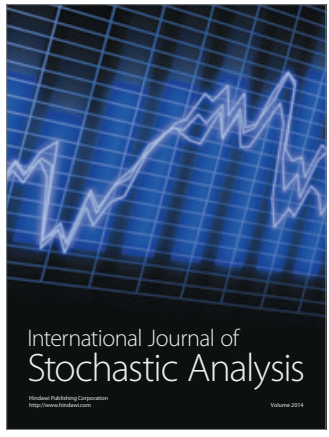

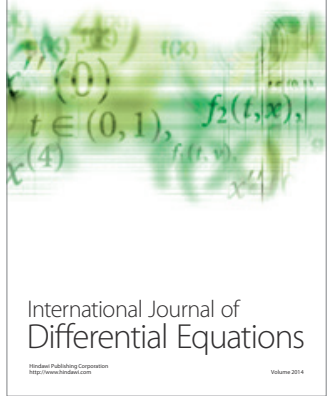
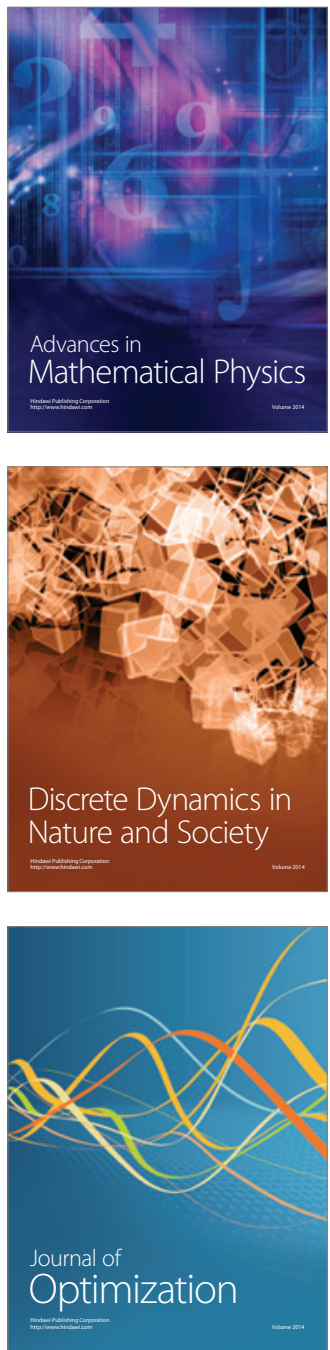